Research Article

\title{
Development and Characterization of Stingless Bee Propolis Properties for the Development of Solid Lipid Nanoparticles for Loading Lipophilic Substances
}

\author{
Putthiporn Khongkaew $\mathbb{i D}^{1}$ and Watcharaphong Chaemsawang ${ }^{2}{ }^{2}$ \\ ${ }^{1}$ Department of Pharmacognosy and Pharmaceutical Chemistry, Faculty of Pharmaceutical Science, Burapha University, \\ 169 Long-Hard Bangsaen Road, Saen Sook Sub-District, Mueang District, Chonburi 20131, Thailand \\ ${ }^{2}$ Department of Pharmaceutical Technology, Faculty of Pharmaceutical Science, Burapha University, \\ 169 Long-Hard Bangsaen Road, Saen Sook Sub-District, Mueang District, Chonburi 20131, Thailand
}

Correspondence should be addressed to Watcharaphong Chaemsawang; watcharaphong@go.buu.ac.th

Received 22 December 2020; Revised 17 May 2021; Accepted 19 May 2021; Published 28 May 2021

Academic Editor: Wen-Cheng Chen

Copyright ( $\odot 2021$ Putthiporn Khongkaew and Watcharaphong Chaemsawang. This is an open access article distributed under the Creative Commons Attribution License, which permits unrestricted use, distribution, and reproduction in any medium, provided the original work is properly cited.

\begin{abstract}
Stingless bees are insects which are popularly bred by agriculturists in the eastern region of Thailand for the pollination of their orchards. The products from stingless bee breeding include bee honey and bee propolis. The objective of this experiment is to study the possibility of developing stingless bee propolis wax into solid lipid nanoparticles (SLN) by the comparison of five surfactants (Brij 721, Cremophor WO 7, Myrj 52, Poloxamer 188, and Tween 80). Each surfactant is used at three concentrations: $10 \%, 20 \%$, and $30 \%$. A master formula is selected according to the following: physical features, particle size, zeta potential, and entrapment. The results showed that Brij 721 and Myri 52 at 20\% can be used in preparing SLN and have good preservation properties for vitamin E (size: $451.2 \mathrm{~nm}$ and $416.8 \mathrm{~nm}$, zeta potential: - 24.0 and - 32.7; \% EE: 92.32\% and 92.00\%, resp.). Therefore, they are further developed by adding the following drugs at low solubility: curcumin, ibuprofen, and astaxanthin. It is found that a formula using the surfactants Brij 721 and Myrj 52 at 20\% have similar drug entrapment. The entrapment study involves curcumin $82 \%$, ibuprofen $40 \%$, and astaxanthin $67 \%$. Moreover, the cytotoxicity test of blank solid lipid nanoparticle found no toxicty in fibroblast cell line (CRL-2522). Therefore, from this study, it is determined that stingless bee propolis wax has the potential to be developed to provide more efficient SLN in the future.
\end{abstract}

\section{Introduction}

Solid lipid nanoparticles (SLN) have been an interesting pharmaceutical delivery system since 1990. The system is composed of wax with a solid status at room temperature and body temperature, a surfactant, and water, creating lipid nanoparticles with features including a solid matrix [1]. There are important drugs spread throughout. In terms of popularity, particle size is mostly in the range of $50-500$ nanometers [2-4]. In general, solid wax is used at $0.1-30 \% \mathrm{w} /$ $\mathrm{w}$ in formulas. Wax with a melting point higher than $40^{\circ} \mathrm{C}$ is popularly used because the product will be stable at room temperature and not melt easily. Particularly, pharmaceutical products transported to tropical countries use such wax.
More than one type of wax can be used, such as triglyceride, Glyceryltristearate (Dynasan $\left.{ }^{\circledR} 118\right), \quad$ Glycerylbehenate (Compritol ${ }^{\circledR} 888$ ATO), stearic acid, carnauba wax, or rice bran wax. However, the SLN is found to be problematic from the wax used, such as cases where the wax has a lower ability in active substance loading. There are also drugs pushed out from the system after storage for some time. Thus, studies to develop types of wax remain ongoing continuously. Stingless bees are insects that are simply bees without a sting, so they are popular for breeding to pollinate orchards, especially in the eastern region of Thailand. These bees are bred in large numbers and, apart from having no stinger, their habits for finding nectar are different from those of Indian hive bees or rock bees. That is, the stingless bee finds nectar not so far 
away, usually within 100-200 meters, and it swarms to flowers indiscriminately. The benefits of stingless bees are honey from the hive, beeswax, and propolis, which is a resinous substance gathered by stingless bees from natural sources such as broken bark, leaf buds, flower nectar, and pollen grain. Therefore, the color and composition of the wax are different according to the area. Generally, the components of propolis are different according to the source. Mostly, they include resin, vegetable balsam $50 \%$, wax $30 \%$, essential oil and aromatic oil $10 \%$, pollen $5 \%$, and other substances 5\% [5-7]. Propolis gathered by stingless bees is mixed with saliva and beeswax, which makes its properties similar to those of aromatic glue, solid and brittle. When heated, it becomes sticky and soft. It is used by stingless bees for nesting and repairing their hives. Propolis is used for plugging broken nests. The study of propolis reveals that the substance from propolis extracted with alcohol provides pharmacological action, such as bacteriostatic effects and antioxidant activity.

Based on the previously mentioned information concerning the benefits of propolis, honey in the industry is separated from the honeycomb and the bee propolis is leftover from the industry. Still, villagers will use the remaining beeswax to make daily-use products, such as candles. Nonetheless, it is still not enough for the high amount of residual wax. In this research, a study was conducted to further develop residual wax for medicinal use to increase drug efficiency and reduce the amount of waste in industries, which is expected from the wax properties. It should be used as a replacement for synthesis wax in the future for the development of a drug delivery system.

\section{Materials and Methods}

Stingless bee propolis was collected from fruit gardens in Chanthaburi, Thailand. Ethanol, methanol, and hexane were purchased from Merck Millipore. Brij 721 was purchased from Croda. Poloxamer 188, Myrj 52, tocopherol acetate, and curcumin were purchased from Sigma Aldrich. Cremophor WO7, Tween 80, and ibuprofen were purchased from PC Drug and Chemical. Astaxanthin was kindly provided by Fuji Chemical. Dulbecco's Modified Eagle's Medium (DMEM) was purchased from Biowest. Fetal bovine serum (FBS), L-glutamax, and antibiotic/antimycotic were purchased from Invitrogen.

2.1. Stingless Bee Wax Extract. Stingless bee propolis was macerated in hexane for 24 hours, after which the extract was filtered with a sheet cloth. The extract was evaporated into concentrate by using a rotary evaporator machine. The wax was then evaporated using a water bath until all the hexane evaporated, followed by storing in a light brown bottle in a refrigerator at $4-8^{\circ} \mathrm{C}$ until use.

2.2. Characterization of Components of Stingless Bees Wax with GC-MS. The substance was dissolved in n-hexane (nHexane; Gas Chromatography MS SupraSolv ${ }^{\circledR}$ Grade, 1.00795 EMD Millipore, Merck). Then, the sample was filtered through a syringe filter (PTFE membrane, hydrophilic type, $0.45 \mu \mathrm{m}$ ) before injection for analysis into GCMS TRACE 1300, where it adhered to an automated liquid sampling set called a TriPlus RSH Autosampler and also to a tandem mass spectrometer called Triple Quadrupole Mass Spectrometer, model: TSQ 8000 Evo Thermo Scientific. The column used for chromatography was TR5-MS (5\% phenyl methyl polysiloxane; $30 \mathrm{~m} \times 0.25 \mathrm{~mm}$ ID, $0.25 \mu \mathrm{m}$ film thickness, Thermo Fisher Scientific). The amount of injection was $1 \mu \mathrm{l}$ split $1: 10$ temp injector of $300^{\circ} \mathrm{C}$. For the temperature setting program of the column oven, the initial value was $80^{\circ} \mathrm{C}$, which was maintained for two minutes, after which it was increased to $350^{\circ} \mathrm{C}$ at a speed of $5^{\circ} \mathrm{C} /$ minute and maintained for 10 minutes. The total time of analysis was 66 minutes per analysis. Carrier gas or helium was used under a flow rate of $1 \mathrm{~mL} / \mathrm{minute}$. For the conditions of mass spectrometry, mass ionization had an electron impact of $70 \mathrm{eV}$. The temperature of the transfer line used was $300^{\circ} \mathrm{C}$, and the temperature of the ion source was $250^{\circ} \mathrm{C}$. Data for the mass spectrum were collected in a full scan MS mode. The mass range was between 50 and 750 amu at a scan time (dwell time) of 0.2 seconds.

2.3. Characterization of Wax with FTIR and DSC. The four types of wax were as follows: (1) bee wax from the breeding stingless bee; (2) synthesized bee wax; (3) rice branch wax; (4) cholesterol. Uniqueness was analyzed with FTIR NICOLET 6700 and tested with Differential Scanning Calorimeters (DSC) (METTLER TOLEDO) by increasing the temperature by $10^{\circ} \mathrm{C} /$ minute using temperatures from $0^{\circ} \mathrm{C}$ to $400^{\circ} \mathrm{C}$.

2.4. Preparation of Solid Lipid Nanoparticles Using Stingless Bee Wax. Each surfactant was used at three concentrations: $10 \%, 20 \%$, and $30 \%$. The surfactants were as follows: Brij 721 , Myrj 52, Tween 80, Poloxamer 188, and Cremophor WO7.

In formula development, the first step of SLN development used tocopherol acetate (vitamin E) as a model drug. The preparation method was a microemulsion technique by mixing excipients, as in Table 1, followed by melting in a water bath. The water phase was poured into the oil phase and then blended with a homogenizer at a speed of $10,000 \mathrm{rpm}$ for 30 minutes. After that, a microemulsion is immediately soaked in an ice bath to solidify. Then, proper formulas are selected to study the experiment in loading with other drugs, such as curcumin, ibuprofen, and astaxanthin.

2.5. Characteristics of Solid Lipid Nanoparticles. The particle size and surface charge are measured using a Nanosizer Series Zetasizer, while the characteristics were evaluated with a scanning electron microscope (SEM).

2.6. Entrapment Efficiency (\%EE). SLN formulation was centrifuged with ultracentrifuge (Amicon) MW $100 \mathrm{~K}$ at a speed of $10,000 \mathrm{rpm}$ for 30 minutes and then washed with $50 \%$ ethanol and centrifuged two times to remove unloaded drugs. The particles on the membrane filter were dissolved 
TABLE 1: Formulation SLN.

\begin{tabular}{lccc}
\hline & Rx10\% surfactant $(\mathrm{g})$ & $\mathrm{Rx} 20 \%$ surfactant $(\mathrm{g})$ & $\mathrm{Rx} 30 \%$ surfactant $(\mathrm{g})$ \\
\hline Stingless bee wax & 10 & 10 & 10 \\
Ethyl alcohol & 14 & 14 & 14 \\
API & 1 & 1 & 1 \\
Surfactants & 10 & 100 & 30 \\
Water qs. to & 100 & 100 & 100 \\
\hline
\end{tabular}

with methanol to measure the absorbance by using UV spectrophotometry series U-2900UV at tocopherol acetate (vitamin E) with a wavelength of $295 \mathrm{~nm}$. Curcumin was analyzed at a wavelength of $549 \mathrm{~nm}$, ibuprofen at a wavelength of $264 \mathrm{~nm}$, and astaxanthin at a wavelength of $470 \mathrm{~nm}$. Curcumin, ibuprofen, and astaxanthin will be examined with the selected formulation from the information of tocopherol acetate.

2.7. Cell Lines and Cultures. The human fibroblast cells (CRL-2522) were purchased by our institution from American Type Culture Collection (ATCC). Cells were seeded in DMEM medium with $10 \%$ fetal bovine serum containing $1 \%$ antibiotic/antimycotic and cultured at $37^{\circ} \mathrm{C}$ in $5 \% \mathrm{CO} 2$. The culture medium was changed every three days.

2.8. Cell Cytotoxicity. Cell cytotoxicity was assessed using the 3-(4, 5-dimethylthiazol-2-yl)-2, 5-diphenyltetrazolium bromide (MTT) assay. $100 \mu \mathrm{l}$ blank solid lipid nanoparticles prepared from Brij 721 or Myrj 52 were diluted in a cell culture complete medium containing $900 \mu \mathrm{l}$. When $1 \times 105$ fibroblast cells had completed a 24-hour incubation in the 96-well plate, the culture medium was replaced with the particle solution and the cells were incubated for another 24 hours. After the completion of the 24-hour incubation, MTT at $5 \mathrm{mg} / \mathrm{ml}$ concentration was added at $10 \mu \mathrm{l} /$ well and incubated for another four hours at $37^{\circ} \mathrm{C}$ and $5 \% \mathrm{CO}_{2}$, then the extract was drained and DMSO 100\% $100 \mu \mathrm{l} /$ well was added to dissolve the formazan in intracellular. Finally, a microplate reader was used to measure at $570 \mathrm{~nm}$ and evaluate with an inverted microscope.

\section{Results and Discussion}

3.1. Characteristics of Stingless Bee Wax by Fourier Transform Infrared Spectroscopy (FTIR). The FTIR spectrum of stingless bee wax is shown in Figure 1 and Table 2. Stingless bee wax has important points that consist of peaks at the point of 2800-2900, which is the sp3 C-H stretching peak of $\mathrm{CH} 2$ and $\mathrm{CH} 3$. This result represents the functional alkanes or alkyl (C-H) peak at the point of 1400 , which is the sp3 C-H bending peak, while the peak shown at $1670-1780$ is $\mathrm{C}=\mathrm{O}$ stretching, which indicates a carbonyl functional group $(\mathrm{C}=\mathrm{O})$. The results show that stingless bee wax is a major component of fatty acids. Comparison with another type of wax in the FTIR spectrum in Figure 2 shows the consistency of the significant elements of stingless bee wax containing fatty acid as well as other types of wax because it establishes the same peak position, except that the position of the stingless bee wax at 1300 is not found in any other type of wax. It is found that there is a peak of $\mathrm{C}-\mathrm{O}$ of ester in the fatty acid, which is an essential component of the stingless bee wax.

3.2. Characteristics of Stingless Bee Wax by Differential Scanning Calorimeters (DSC). Differential Scanning Calorimeters (DSC) is used to measure the heat flow of the sample when being heated. The physical and chemical changes of the heated sample can be used for patterns of melting and the primary component.

From the results of the DSC thermogram (Figure 3) when compared with the peaks of synthetic bee wax and rice bran wax, there were different melting points compared with stingless bee wax, for which the proportional quantity of fatty acid is also different. Stingless bee wax has a higher melting point, which might be caused by the component of the fatty acids at saturated hydrocarbons [8]. This is an advantage for the lipid particle delivery system because of its superior stability compared with other kinds of wax in the part of the cholesterol, for which the main component is steroids $[9,10]$. The thermal change is different from other substances.

3.3. Characteristics of Stingless Bee Wax by Gas Chromatography-Mass Spectrometry (GC-MS). From the chromatogram of GC-MS (Figure 4), it was found that the major components of stingless bee wax included lipids, that is, long-chain fatty acids in the form of ester (3.24\%), and hydrocarbons with a total length of $39.18 \%$. Cholesterol and triterpenoids were also found from 48.37 minutes, which totaled $42.1 \%$ (Table 3 ). This conformed to the experiment by DSC, from which the thermogram showed that stingless bee wax had two melting point positions. To clarify, the melting point at $58.6^{\circ} \mathrm{C}$ was close to that of cholesterol. The other melting point at around $87-88^{\circ} \mathrm{C}$ was higher than the bee wax and rice bran wax, possibly from the components of the wax, which were mostly saturated hydrocarbons. For this reason, the melting point was higher than the lipids with unsaturated hydrocarbons. Therefore, the advantage of stingless bee wax when it is prepared as a solid lipid nanoparticle is that it has more stability than other natural lipids because it is not oxidized as easily. The arrangement of crystals was also lower than the unsaturated lipids. Moreover, the triterpenoids that were found conformed to the previous study, which discovered that propolis from natural bees had an antibacterial activity [10]. Substances found in stingless bee propolis $\beta$-amyrone, ester derivatives, and 9, 19-cyclo-9 $\beta$ lanost-24-en-3 $\beta$-ol were found to have antioxidation and 


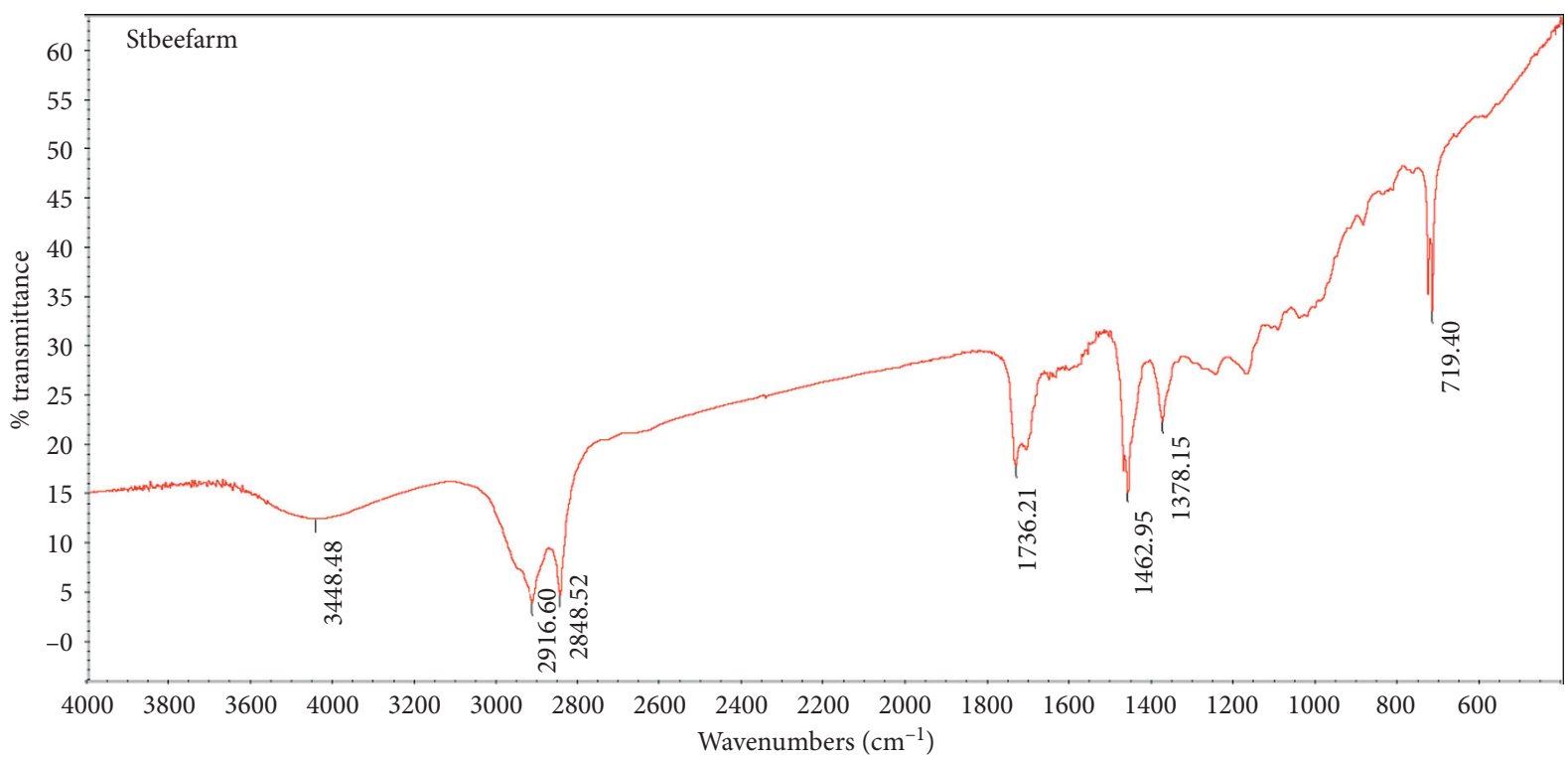

FIGURE 1: The spectrum represents the peak of stingless bees wax.

TABLE 2: FTIR characteristic peaks of stingless bee wax.

\begin{tabular}{|c|c|c|}
\hline Wave number ${ }^{-1}$ & Appearance & Group \\
\hline 1462.95 & Weak & $\mathrm{C}-\mathrm{H}$ bending \\
\hline 1736.21 & Strong & $\mathrm{C}=\mathrm{O}$ stretching \\
\hline $2000-2500$ & Weak & Overtone of aromatic compound \\
\hline $2916.60,2848.52$ & Strong & C-H stretching \\
\hline 3448.48 & Strong & O-H stretching \\
\hline
\end{tabular}

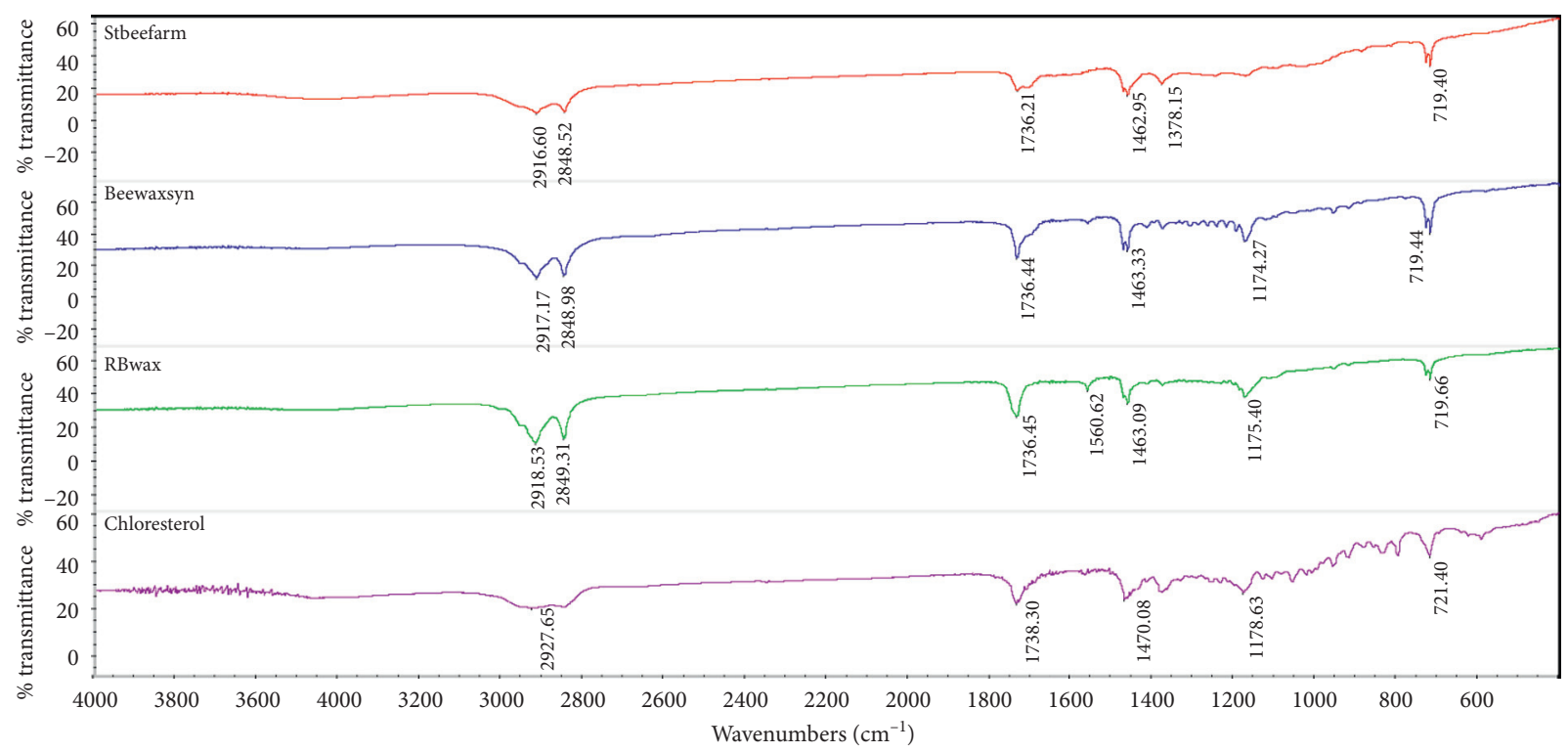

FIgURE 2: The FTIR spectra shows peaks of stingless bee wax (red), synthetic bee wax (blue), rice bran wax (green), and cholesterol (purple).

antibacterial properties [11-13] Hence, applying prepared solid lipid nanoparticles for medical or cosmetic use would tend to be beneficial.
3.4. Characteristics of Stingless Bee Wax Solid Nanoparticles. From the preparation of the SLN particles with five types of surfactants, it was found that Cremophor WO 7 could not 


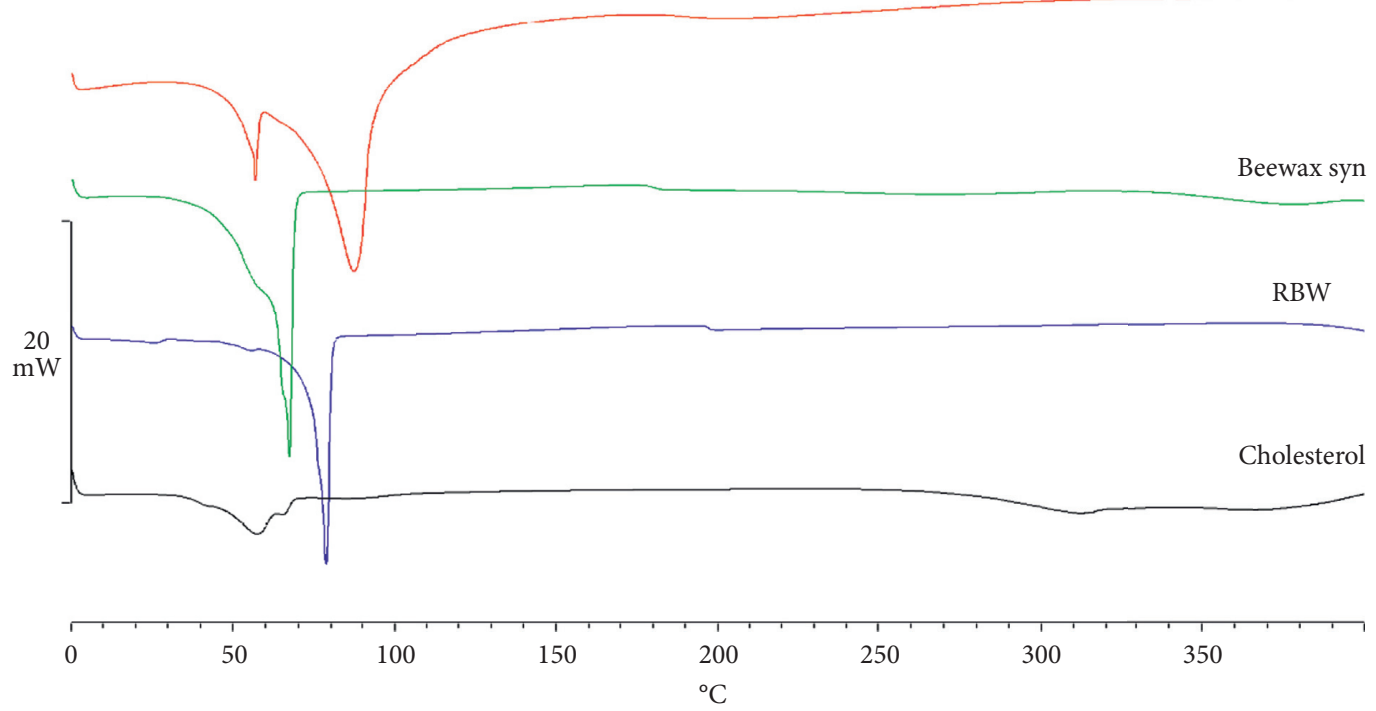

Figure 3: Thermogram of stingless bee wax, synthetic bee wax, rice bran wax, and cholesterol, respectively, by Differential Scanning Calorimeter.

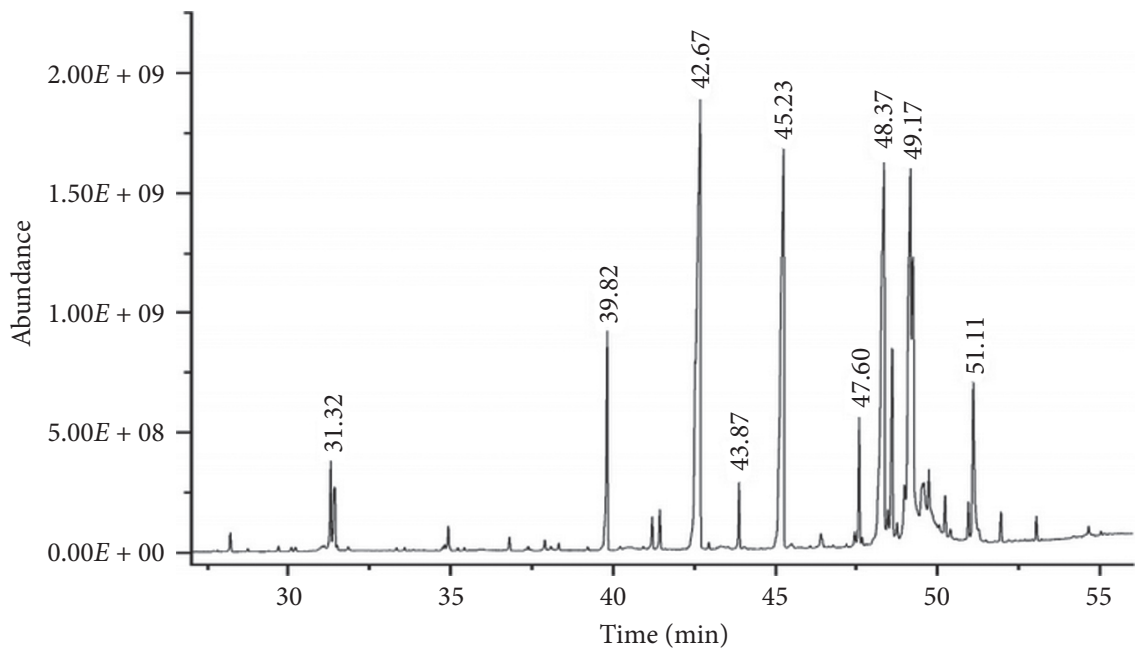

FIGURE 4: The GC-MS chromatrogram of stingless bee wax.

prepare SLN. The cream was very thick and unable to separate the particles, thus resulting in the inability to collect any particles for the physical quality assessment. Regarding the other surfactants, Brij 721, Myrj 52, Tween 80, and Poloxamer 188 (Table 4 and Figure 5), they showed similar results in terms of type and concentration per particle size. At a $10 \%$ concentration, the particle size is smaller. The ability of surfactant to reduce surface tension is not yet complete, thus creating particles that are very small to very large. Moreover, the entrapment of vitamin $\mathrm{E}$ is incomplete. For instance, $10 \%$ of Poloxamer 188 has lower entrapment efficiency than using concentrations of $20 \%$ and $30 \%$. As such, this represented incomplete particle formation that affected less drug entrapment. However, when the concentration of the surfactants was increased, the particle size became slightly larger, even though the particle result was improved due to the reduction of surface tension and the higher entrapment efficiency. However, the particle size increased when using nonionic surfactants in the concentration range. The research of Hazzah et al. [14] can explain the phenomenon that occurs when increasing the concentration of the surfactant, where the particle size would be larger. The force of the hydrocarbon chain of the surfactant attracted Van Der Waals forces among the hydroxyl group in the hydrocarbon chain, causing the wax droplets to coalesce into large droplets before solidifying. Alternatively, the particle would become smaller again when the concentration of the surfactant increased. The mechanism of the reduced surface tension is strong enough to cover the thermodynamic force. Therefore, the particles can remain in their shape, even with a high surface area, resulting in smaller and more stable particles [15]. Although the high 
TABLE 3: Tentative compounds found in stingless bee wax.

\begin{tabular}{|c|c|c|}
\hline$t R(\min )$ & Compound & Relative abundance (\%) \\
\hline $30.12-31.86$ & $\begin{array}{c}\text { Fatty acid ester } \\
\text { Linoleic acid, methyl ester } \\
\text { Linolenic acid, methyl ester } \\
\text { cis-9, cis-12-linoleic acid } \\
\text { Linoleic acid, ethyl ester } \\
\text { Oleic acid, ethyl ester } \\
\text { Elaidic acid, ethyl ester } \\
\text { Stearic acid, ethyl ester }\end{array}$ & 3.24 \\
\hline 39.82 & Hepta cosine & 4.01 \\
\hline 42.67 & Nonacosane & 20.01 \\
\hline 45.23 & Hentriacontane & 15.16 \\
\hline 48.37 & Lupen-3-one & 15.12 \\
\hline 48.62 & $\beta$-Amyrone & 3.68 \\
\hline 49.17 & 9, 19-Cyclo-9 $\beta$-lanost- 24 -en- $3 \beta$-ol & 11.36 \\
\hline 49.26 & $\beta$-Amyrin 3-acetate & 7.83 \\
\hline \multirow[t]{3}{*}{51.11} & 23-(Phenylsulfanyl) lanosta-8, 24-dien-3-ol & 4.11 \\
\hline & Others & 6.31 \\
\hline & Total unknown & 9.17 \\
\hline
\end{tabular}

TABLE 4: The comparison of the particle size of zeta potential and \% entrapment efficiency of the formation containing vitamin E ( $n=3$ ); ZP: zeta potential.

\begin{tabular}{lccr}
\hline Surfactant (\%) & Size & ZP & \% EE \\
\hline Brij 721 & & & \\
10 & $461.4 \pm 17.82$ & $-26.0 \pm 1.36$ & $96.62 \pm 0.00$ \\
20 & $451.2 \pm 6.58$ & $-24.0 \pm 0.51$ & $92.32 \pm 0.19$ \\
30 & $292.1 \pm 23.60$ & $-13.3 \pm 0.45$ & $74.59 \pm 0.13$ \\
\hline Cremophor WO 7 & & & N/A \\
10 & N/A & N/A & N/A \\
20 & N/A & N/A & N/A \\
30 & N/A & N/A & \\
Myrj 52 & & & \\
10 & $366.4 \pm 7.73$ & $-28.4 \pm 0.41$ & \\
20 & $416.8 \pm 44.61$ & $-32.7 \pm 0.96$ & \\
30 & $366.8 \pm 3.09$ & $-27.5 \pm 1.12$ & \\
\hline Poloxamer 188 & & & \\
10 & $466.9 \pm 64.20$ & $-30.6 \pm 1.13$ & \\
20 & $759.3 \pm 88.52$ & $-32.9 \pm 0.15$ & \\
30 & $388.0 \pm 1.99$ & $-32.2 \pm 0.35$ & \\
\hline Tween 80 & & & \\
10 & $357.8 \pm 39.97$ & $-27.3 \pm 2.09$ & \\
20 & $756.6 \pm 61.24$ & $-24.1 \pm 0.45$ & \\
30 & $312.2 \pm 17.11$ & $-25.2 \pm 3.11$ & \\
\hline
\end{tabular}

concentration of the surface tension minimized the particles, the percentage of the entrapment was also reduced. The decrease in entrapment is because of the very high concentration of surfactants as solubilizers; hydrophobic agents can be soluble with micellar mechanisms. Therefore, some drugs dissolve in the surfactant, such as micelle, and disperse in the aqueous phase, but do not dissolve in the oil phase $[16,17]$. A drop of wax is created when solidified into particles with lower drug content than it should be. From the experimental results of the vitamin E loading SLN preparation (Table 5), a suitable concentration of the surfactant was $20 \%$ with the maximum percentage of entrapment, when considering the parameters and physical characteristics, as previously mentioned. Regarding the type of surfactants, Brij 721 and Myrj 52 offered the best particle properties with a smaller size and higher entrapment. It was also found that Brij 721 and Myrj 52 had a linear molecule structure, and the number of carbon chains was lower than Tween 80, and Poloxamer 188 (Figure 6). Tween 80 had a long carbon side chain, which had a branch chain type, while Poloxamer 188 also had a linear polymer, but the molecular weight was higher. Hence, the structure is bent into the $U$ shape when poloxamer 188 forms the micelles, with the hydrophilic part facing outwards [18]. This was the reason why the molecules of Brij 721 and Myrj 52 were small and could cover the particle's surface with more stability. 


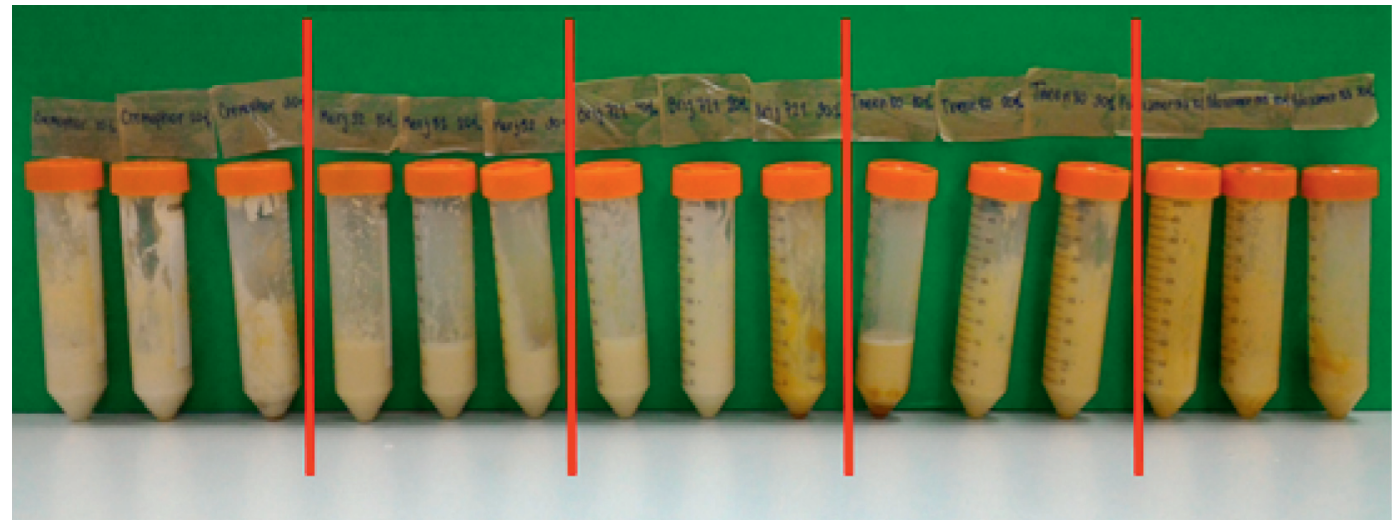

(a)

(b)

(c)

(d)

(e)

Figure 5: Physical properties of each surfactant. (a) Cremophor WO7, (b) Myrj 52, (c) Brij 721, (d) Tween 80, and (e) Poloxamer 188 (surfactant concentration $10 \%, 20 \%$, and $30 \%$ ).

TABLE 5: The comparison of the particles of zeta potential and \% entrapment efficiency $(n=3)$.

\begin{tabular}{lccr}
\hline & Size & ZP & $\%$ EE \\
\hline Curcumin & & & \\
Brij 721, 20\% & $426.3 \pm 6.93$ & $-28.0 \pm 0.42$ & $92.37 \pm 0.00$ \\
Myrj 52, 20\% & $445.5 \pm 14.31$ & $-31.5 \pm 0.49$ & $74.32 \pm 0.14$ \\
\hline Ibuprofen & & & \\
Brij 721, 20\% & $443.3 \pm 6.93$ & $-19.9 \pm 0.42$ & $40.68 \pm 0.00$ \\
Myrj 52, 20\% & $442.2 \pm 14.31$ & $-37.8 \pm 0.49$ & $40.97 \pm 0.14$ \\
\hline Astaxanthin & & & \\
Brij 721, 20\% & $407.5 \pm 6.93$ & $-25.7 \pm 0.42$ & $65.33 \pm 0.00$ \\
Myrj 52, 20\% & $451.3 \pm 14.31$ & $-33.6 \pm 0.49$ & $68.20 \pm 0.14$ \\
\hline
\end{tabular}

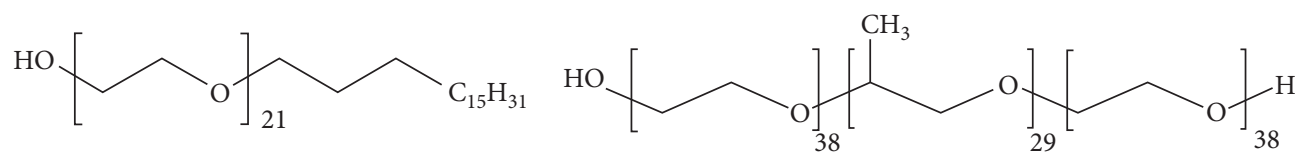

(a)

(b)

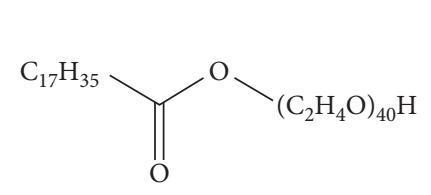

(c)

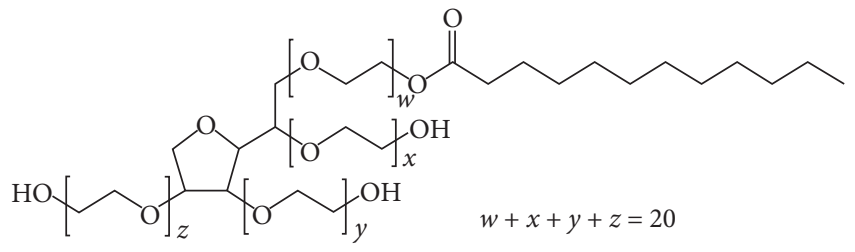

(d)

Figure 6: Chemical structure of each surfactant. (a) Brij 721, (b) Poloxamer 188, (c) Myrj 52, and (d) Tween 80.

Concerning microemulsion preparation using the surfactant with small particles or cosurfactant, the microemulsion remained more stable than using the surfactant with large particles [15, 19-22]. Figure 7 shows the morphology of SLN from both types of surfactants (Brij 721; Myrj 52), which had spherically-shaped particles under SEM. For the results of zeta potential values, nanometer particle size typically requires a charge of -30 or $+30 \mathrm{mv}$ [23-25] and above to maintain particle stability. Zeta potential values closer to zero will result in particle aggregation. The findings showed a negative charge of the particles due to the carboxylic group in the fatty acid found in wax. The results found that nonionic surfactant will not significantly affect the value of zeta potential.

The research results show that the formulations prepared from Brij 721, 20\%, had an average smaller particle size than Myrj 52, 20\%, but the findings were similar when evaluating the $\%$ entrapment efficiency (Table 4 ). The results show that curcumin has the highest drug entrapment because of its high molecular weight; the higher wax solubility creates a better possibility for wax solubility [26]. In addition, ibuprofen has less entrapment efficiency because its chemical 


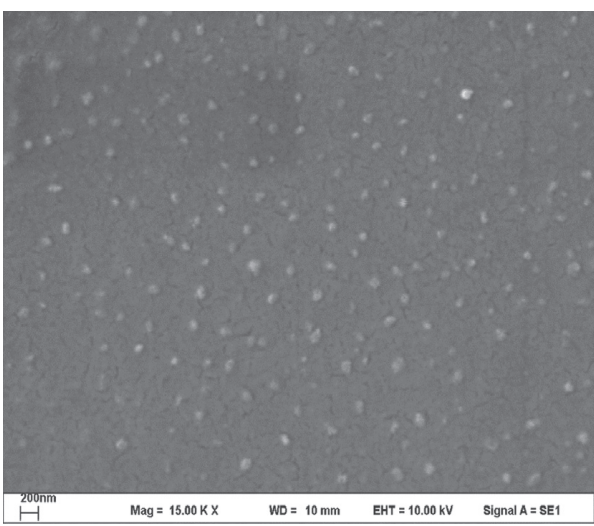

(a)

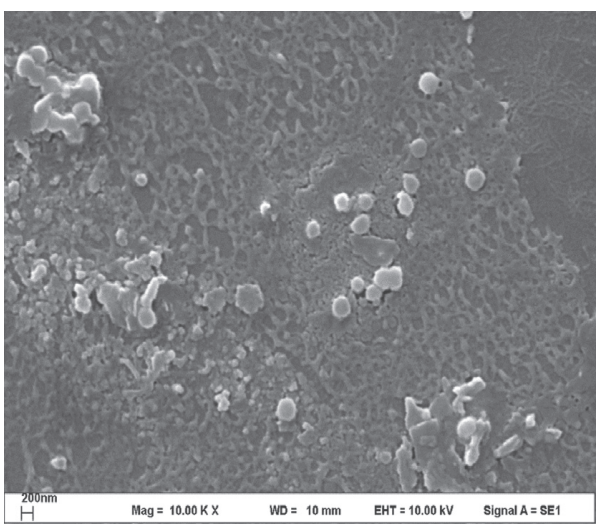

(b)

Figure 7: The photographs of the scanning electron microscope. (a) Brij 721, 20\%; (b) Myrj 52, $20 \%$.

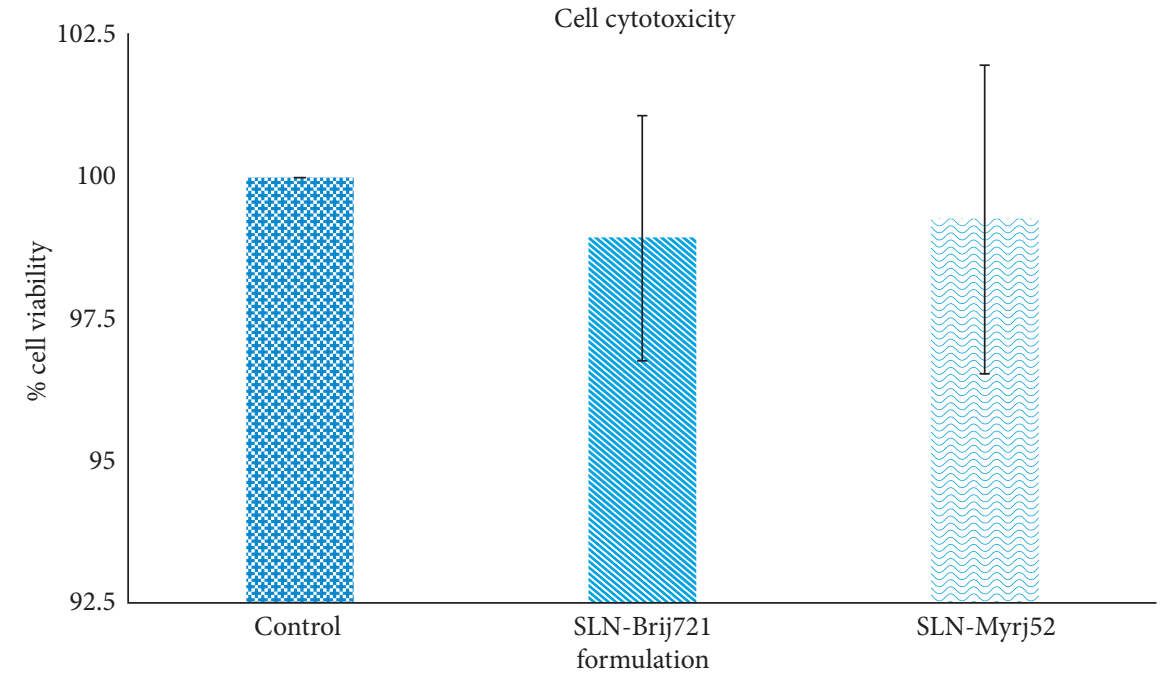

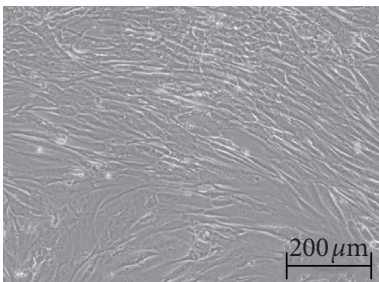

(a)

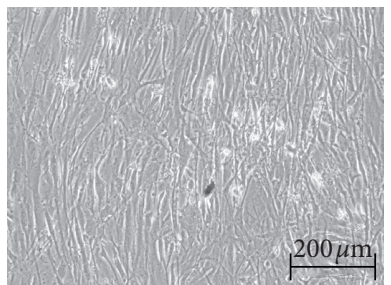

(b)

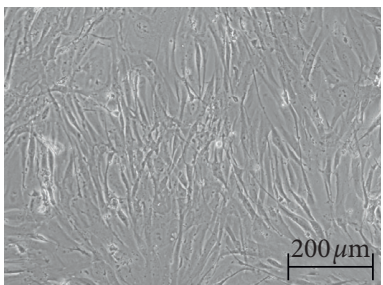

(c)

Figure 8: Cell cytotoxicity. (a) Control, (b) blank SLN from Brij 721, and (c) blank SLN from Myrj 52.

structures have polar functional groups such as carboxylic groups. Therefore, the drug may be easily pushed out of liquid wax during wax solidification $[27,28]$.

3.5. Cytotoxicity. The cytotoxicity of SLN from stingless bee propolis was determined using the MTTassay method on the fibroblast cell line. Data showed both SLN prepared from Brij 721 and Myrj 52 had no significant difference in toxicity when compared with the control group or the preparation from Brij and Myrj (Figure 8). Observation under a microscope to determine particles cannot be done because the size of SLN is too small. Furthermore, another research has also found that the SLN delivery system is safe for human cells. It also found that fibroblast cells can deliver SLN into a cell by endocytosis pathway before degrading with lysozyme to release drugs $[29,30]$. This might also be the reason why the SLN particles are invisible outside the cells under a microscope. The previous research by Karting et al. [31], concerning delivery of glucocorticoid by SLN, shows that SLN can increase the permeation of glucocorticoids by three times compared with the control. Furthermore, the survival rate of the cells is $94.5 \%$ after 18 hours of incubation. The results conformed to this research. Moreover, the SLN 
system reduced skin irritation that had the irritation quality in the animal test [32]. From the previous research, it can be concluded that the SLN delivery system is safe and could be developed for use on the human body. Furthermore, it increases the chance of the absorption rate and decreases the toxins from the medicine. This observation provides support for the development of solid lipid nanoparticles from stingless bee propolis for future pharmaceutical purposes.

\section{Conclusion}

According to the research results, it was found that the wax of stingless bees could be developed and used as a medicine delivery system. The study found that the components in stainless bee wax include fatty acid and terpenoids. The terpenoids were reported for their antimicrobial activity. Therefore, it could be studied or developed further for pharmaceutical use in the treatment of localized infections. In addition, the physicochemical test from DSC found that stingless bee wax had high physicochemical stability compared with other types of wax, resulting in more stability under long-term storage. Lastly, the toxicity test showed no toxicity in the fibroblast cells, in the prepared formula from either Brij 721 or Myrj 52. Thus, the delivery system of a solid lipid nanoparticle could be used in real situations in the future, by either transdermal drug delivery or oral medication. However, this research study is only a basic evaluation of the characteristics of stingless bee wax. Further studies must be performed in greater depth to assess its stability and development of the delivery system.

\section{Data Availability}

Data used in this study are available upon request.

\section{Conflicts of Interest}

The authors declare that they have no conflicts of interest.

\section{Acknowledgments}

The authors would like to acknowledge the support of the Faculty of Pharmaceutical Science at Burapha University, which provided research equipment that allowed conducting this study.

\section{References}

[1] M. C. Teixeira, C. Carbone, and E. B. Souto, "Beyond liposomes: recent advances on lipid based nanostructures for poorly soluble/poorly permeable drug delivery," Progress in Lipid Research, vol. 68, pp. 1-11, 2017.

[2] A. Garcês, M. H. Amaral, J. M. Sousa Lobo, and A. C. Silva, "Formulations based on solid lipid nanoparticles (SLN) and nanostructured lipid carriers (NLC) for cutaneous use: a review," European Journal of Pharmaceutical Sciences, vol. 112, pp. 159-167, 2018.

[3] M. D. Joshi and R. H. Müller, "Lipid nanoparticles for parenteral delivery of actives," European Journal of Pharmaceutics and Biopharmaceutics, vol. 71, no. 2, pp. 161-172, 2009.
[4] J. Pardeike, A. Hommoss, and R. H. Müller, "Lipid nanoparticles (SLN, NLC) in cosmetic and pharmaceutical dermal products," International Journal of Pharmaceutics, vol. 366, no. 1-2, pp. 170-184, 2009.

[5] F. C. Biluca, J. S. de Gois, M. Schulz et al., "Phenolic compounds, antioxidant capacity and bioaccessibility of minerals of stingless bee honey (Meliponinae)," Journal of Food Composition and Analysis, vol. 63, pp. 89-97, 2017.

[6] M. K. Choudhari, S. A. Punekar, R. V. Ranade, and K. M. Paknikar, "Antimicrobial activity of stingless bee (Trigona sp.) propolis used in the folk medicine of Western Maharashtra, India," Journal of Ethnopharmacology, vol. 141, no. 1, pp. 363-367, 2012.

[7] P. de Lima Morais da Silva, L. S. de Lima, Í. K. Caetano, and Y. R. Torres, "Comparative analysis of the volatile composition of honeys from Brazilian stingless bees by static headspace GC-MS," Food Research International, vol. 102, pp. 536-543, 2017.

[8] W. F. Tinto, T. O. Elufioye, and J. Roach, "Waxes pharmacognosy," in Pharmacognosy, S. Badal and R. Delgoda, Eds., Academic Press, Boston, MA, USA, pp. 443-455, 2017.

[9] G. A. Burdock, "Review of the biological properties and toxicity of bee propolis (propolis)," Food and Chemical Toxicology, vol. 36, no. 4, pp. 347-363, 1998.

[10] N. Zabaiou, A. Fouache, A. Trousson et al., "Biological properties of propolis extracts: something new from an ancient product," Chemistry and Physics of Lipids, vol. 207, pp. 214-222, 2017.

[11] Y.-C. Su, C.-T. Liu, Y.-L. Chu, R. Raghu, Y.-H. Kuo, and L.-Y. Sheen, "Eburicoic acid, an active triterpenoid from the fruiting bodies of basswood cultivated antrodia cinnamomea, induces ER stress-mediated autophagy in human hepatoma cells," Journal of Traditional and Complementary Medicine, vol. 2, no. 4, pp. 312-322, 2012.

[12] X. Niu, H. Yao, W. Li et al., " $\delta$-amyrone, a specific inhibitor of cyclooxygenase-2, exhibits anti-inflammatory effects in vitro and in vivo of mice," International Immunopharmacology, vol. 21, no. 1, pp. 112-118, 2014.

[13] E. Michalakea, K. Graikou, N. Aligiannis et al., "Isolation and structure elucidation of secondary metabolites of two Greek endemic Inula species. Biological activities," Phytochemistry Letters, vol. 31, pp. 155-160, 2019.

[14] H. A. Hazzah, R. M. Farid, M. M. A. Nasra, W. A. Hazzah, M. A. El-Massik, and O. Y. Abdallah, "Gelucire-based nanoparticles for curcumin targeting to oral mucosa: preparation, characterization, and antimicrobial activity assessment," Journal of Pharmaceutical Sciences, vol. 104, no. 11, pp. 3913-3924, 2015.

[15] C. M. Keck, A. Kovačević, R. H. Müller, S. Savić, G. Vuleta, and J. Milić, "Formulation of solid lipid nanoparticles (SLN): the value of different alkyl polyglucoside surfactants," International Journal of Pharmaceutics, vol. 474, no. 1-2, pp. 33-41, 2014.

[16] M. Ghadiri, S. Fatemi, A. Vatanara et al., "Loading hydrophilic drug in solid lipid media as nanoparticles: statistical modeling of entrapment efficiency and particle size," International Journal of Pharmaceutics, vol. 424, no. 1-2, pp. 128-137, 2012.

[17] T. Helgason, T. S. Awad, K. Kristbergsson, D. J. McClements, and J. Weiss, "Effect of surfactant surface coverage on formation of solid lipid nanoparticles (SLN)," Journal of Colloid and Interface Science, vol. 334, no. 1, pp. 75-81, 2009.

[18] W. Chaemsawang, "The effect of okra (abelmoschus esculentus (L.) moench) seed extract on human cancer cell lines 
delivered in its native form and loaded in polymeric micelles," International Journal of Biomaterials, vol. 2019, Article ID 9404383, 13 pages, 2019.

[19] K. Karn-orachai, S. M. Smith, S. Saesoo et al., "Surfactant effect on the physicochemical characteristics of $\gamma$-oryanolcontaining solid lipid nanoparticles," Colloids and Surfaces A: Physicochemical and Engineering Aspects, vol. 488, pp. 118$128,2016$.

[20] A. Wang, L. Chen, F. Xu, and Z. Yan, "Phase behavior of epoxidized soybean oil-based ionic liquid microemulsions: effects of ionic liquids, surfactants, and co-surfactants," Colloids and Surfaces A: Physicochemical and Engineering Aspects, vol. 481, pp. 500-505, 2015.

[21] R. Sripriya, K. Muthu Raja, G. Santhosh, M. Chandrasekaran, and M. Noel, "The effect of structure of oil phase, surfactant and co-surfactant on the physicochemical and electrochemical properties of bicontinuous microemulsion," Journal of Colloid and Interface Science, vol. 314, no. 2, pp. 712-717, 2007.

[22] M. M. Mojahedian, S. Daneshamouz, S. M. Samani, and A. Zargaran, "A novel method to produce solid lipid nanoparticles using n-butanol as an additional co-surfactant according to the o/w microemulsion quenching technique," Chemistry and Physics of Lipids, vol. 174, pp. 32-38, 2013.

[23] E. Joseph and G. Singhvi, "Multifunctional nanocrystals for cancer therapy: a potential nanocarrier nanomaterials for drug delivery and therapy," in Nanomaterials for Drug Delivery and Therapy, A. M. Grumezescu, Ed., William Andrew Publishing, Norwich, NY, USA, pp. 91-116, 2019.

[24] V. Selvamani, "Stability studies on nanomaterials used in drugs characterization and biology of nanomaterials for drug delivery," in Characterization and Biology of Nanomaterials for Drug Delivery, S. S. Mohapatra et al., Ed., Elsevier, Amsterdam, Netherlands, pp. 425-444, 2019.

[25] N. M. Zuki, N. Ismail, and F. M. Omar, "Evaluation of zeta potential and particle size measurements of multiple coagulants in semiconductor wastewater," AIP Conference Proceedings, vol. 2124, no. 1, Article ID 020036, 2019.

[26] R. Kumar, A. Singh, N. Garg, and P. F. Siril, "Solid lipid nanoparticles for the controlled delivery of poorly water soluble non-steroidal anti-inflammatory drugs," Ultrasonics Sonochemistry, vol. 40, pp. 686-696, 2018.

[27] K. Göke and H. Bunjes, "Drug solubility in lipid nanocarriers: influence of lipid matrix and available interfacial area," International Journal of Pharmaceutics, vol. 529, no. 1-2, pp. 617-628, 2017.

[28] G. Zoubari, S. Staufenbiel, P. Volz, U. Alexiev, and R. Bodmeier, "Effect of drug solubility and lipid carrier on drug release from lipid nanoparticles for dermal delivery," European Journal of Pharmaceutics and Biopharmaceutics, vol. 110, pp. 39-46, 2017.

[29] J. Fritsch, M. J. Buckmaster, and B. Storrie, "Fibroblasts maintain a complete endocytic pathway in the presence of lysosomotropic amines," Experimental Cell Research, vol. 175, no. 2, pp. 277-285, 1988.

[30] V. Karabanovas, Z. Zitkus, D. Kuciauskas, R. Rotomskis, and M. Valius, "Surface properties of quantum dots define their cellular endocytic routes, mitogenic stimulation and suppression of cell migration," Journal of Biomedical Nanotechnology, vol. 10, no. 5, pp. 775-786, 2014.

[31] C. S. Maia, W. Mehnert, and M. Schäfer-Korting, "Solid lipid nanoparticles as drug carriers for topical glucocorticoids," International Journal of Pharmaceutics, vol. 196, no. 2, pp. 165-167, 2000.
[32] J. O. Morales, K. Valdés, J. Morales, and F. OyarzunAmpuero, "Lipid nanoparticles for the topical delivery of retinoids and derivatives," Nanomedicine, vol. 10, no. 2, pp. 253-269, 2015. 